\title{
Prevalencia y características del síndrome metabólico en personal docente y administrativo de Ia UAA
}

\author{
Sandoval-Valdez Darío*, Hernández-González Flavio*, Terrones-Saldívar Ma del Carmen**
}

\begin{tabular}{|c|c|}
\hline 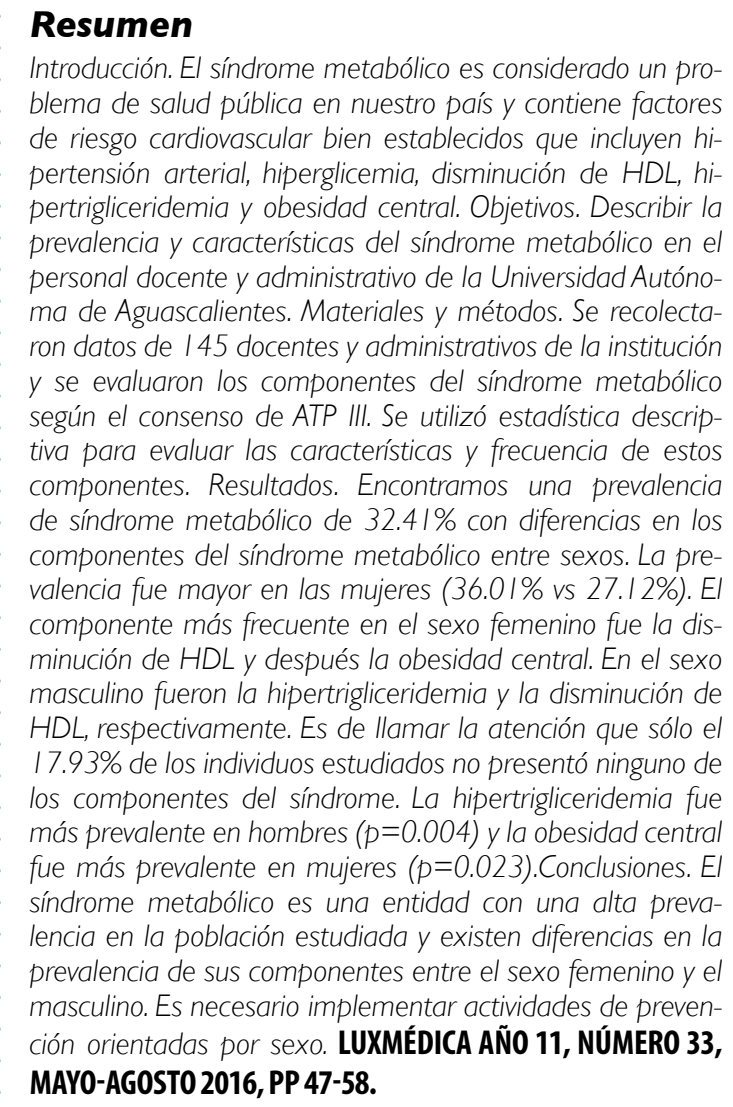 & 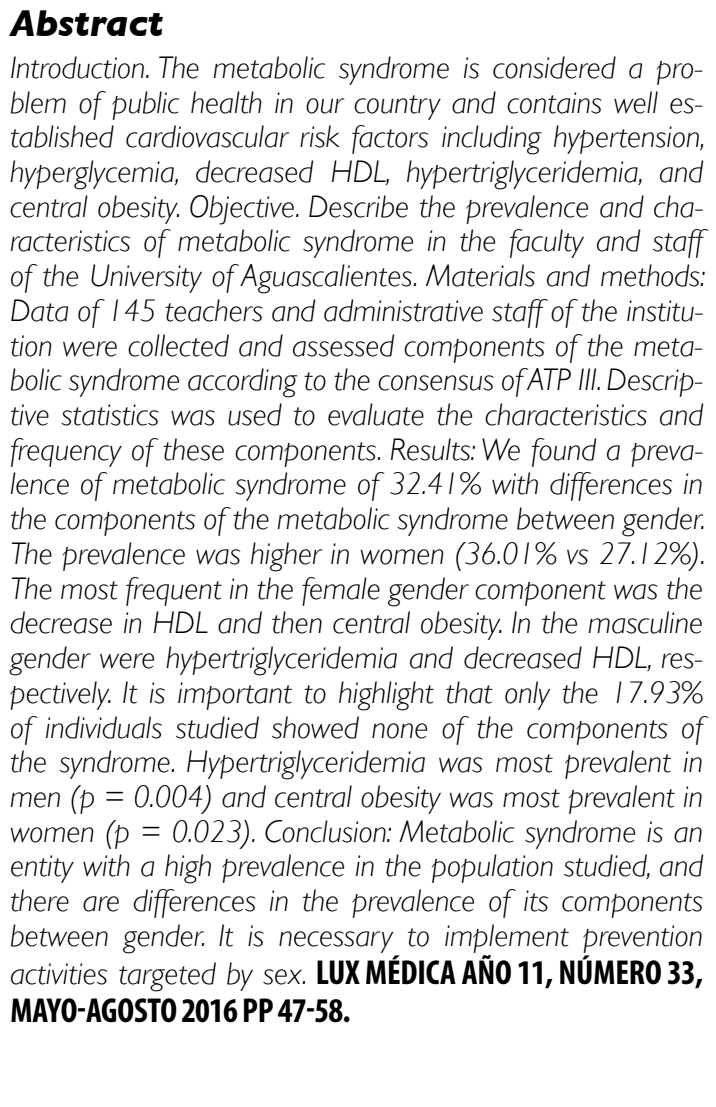 \\
\hline Palabras clave: Síndrome metabólico, dislipidemi & D \\
\hline
\end{tabular}

* Estudiantes del octavo semestre de la carrera de Medicina del Centro de Ciencias de la Salud de la Universidad Autónoma de Aguascalientes.

** Médico Cirujano, especialista en Ginecología y Obstetricia, Maestría en Toxicología y Doctorado en Farmacología. Profesora investigadora del Departamento de Gineco-Obstetricia del Centro de Ciencias de la Salud de la Universidad Autónoma de Aguascalientes.

Fecha de recibido: 10 de diciembre 2015

Fecha de aceptación: 26 de enero 2016

Correspondencia: Dra en C Ma del Carmen Terrones Saldívar. Departamento de Gineco-Obstetricia y Pediatría. Edificio 107 planta alta. Centro de Ciencias de la Salud. Ciudad Universitaria. Universidad Autónoma de Aguascalientes. Avenida Universidad 940. Código postal 20131. Aguascalientes, Ags., México. Teléfono 014499108443. Correo electrónico mcterron@correo.uaa.mx 


\section{Introducción}

El síndrome metabólico es un conjunto de alteraciones metabólicas que incrementan el riesgo cardiovascular. Los componentes del síndrome metabólico son la obesidad, hipertrigliceridemia, disminución de colesterol HDL, hipertensión e hiperglucemia (NCEP-ATP III, 2002)".

En México, es bien conocido el problema de salud que representa la obesidad y los trastornos asociados a ésta. La obesidad, como parte del síndrome metabólico, es un factor de riesgo de enfermedad cardiovascular y otras enfermedades como cáncer ${ }^{2}$, diabetes mellitus tipo $2^{3}$, entre otras. En nuestro país, estas alteraciones metabólicas adquieren una importancia especial pues representan un costo muy elevado para el sistema de salud, pudiendo representar hasta un $25 \%$ del gasto total en salud en el tratamiento integral de la obesidad ${ }^{4}$.

Cada uno de los componentes del síndrome metabólico y todos ellos en conjunto han sido correlacionados con la severidad de cardiopatía isquémica y otras enfermedades cardiovasculares ${ }^{5}$. Es importante reconocer y actuar frente al síndrome metabólico, ya que se ha demostrado que sus componentes son predictores de enfermedad cardiovascular, principalmente de cardiopatía isquémica. ${ }^{6,7}$ Sólo en Aguascalientes, las enfermedades cardíacas como la cardiopatía isquémica y la hipertensión arterial representaron la primera causa de muerte en los años del 2010 al $2014 .^{8}$ Cifras similares existen a nivel nacional. ${ }^{9}$ Se ha estudiado la prevalencia de síndrome metabólico en diferentes poblaciones e incluso se han definido parámetros para éste dependiendo de la población. Se ha estipulado el impacto del síndrome metabólico en el desarrollo de enfermedades cardiovasculares y el impacto de éstas en la mortalidad general de la población mexicana, es por esto que existen diferentes guías clínicas para el tratamiento de sus componentes.

La prevalencia de este síndrome es variable en el mundo sobre todo por la diferencia en los criterios utilizados para definirlo. La encuesta para la evaluación de la salud nacional y nutrición (NHANES) en Estados Unidos ha reportado los factores de riesgo más frecuentes por sexo (figura 1), mostrando la obesidad centrípeta y la hiperglucemia como los componentes más prevalentes en ambos sexos.

Se han realizado estudios de prevalencia en diferentes poblaciones. En lo que respecta a la población mexicana se han realizado estudios de prevalencia del síndrome metabólico. Un estudio de 2014, basado en la Encuesta Nacional en Salud 200610 encontró una prevalencia de $49.8 \%$ (47.5\% - 52.1\%) de síndrome metabólico en la población mexicana mayor de 20 años. Los componentes más frecuentes encontrados fueron HDL baja (76.8\%) y obesidad centrípeta (73.6\%). ${ }^{11}$ En ese estudio, se propuso clasificar al síndrome metabólico según la dislipidemia presentada, en síndrome 


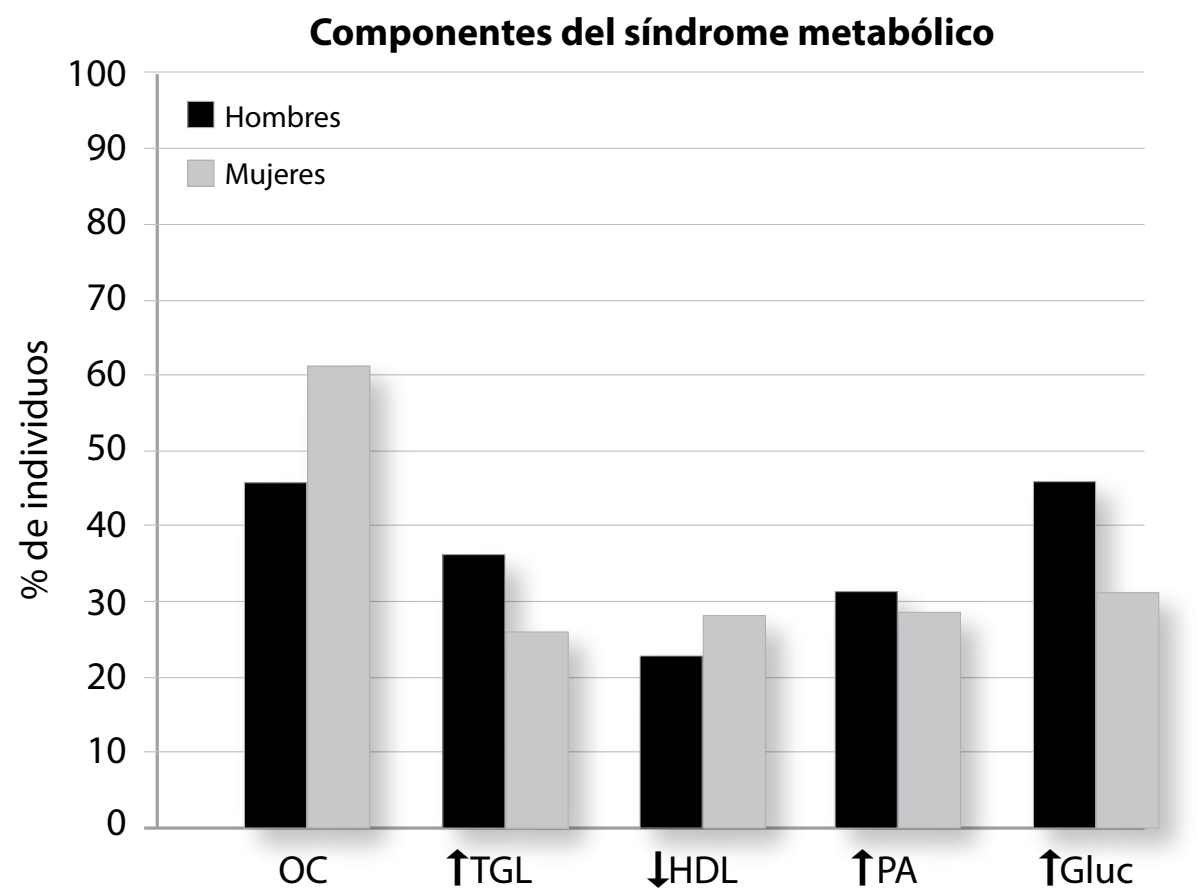

Figura 1. Frecuencia relativa por sexo de los componentes del síndrome metabólico en adultos según la encuesta nacional en salud (NHANES) en EUA. Tomada y modificada de Harrison Principios de Medicina Interna, $19^{a}$ edición. OC: Obesidad central, $\uparrow$ TGL: Hipertrigliceridemia, $\downarrow_{H D L}$ : Disminución de lipoproteínas de baja densidad,

$\uparrow$ PA: Hipertensión, $\uparrow$ Gluc: Hiperglucemia

metabólico con dislipidemia mixta, con disminución de HDL, con hipertrigliceridemia o sin dislipidemia. El presente trabajo tiene por objeto conocer la prevalencia y las características del síndrome metabólico en los trabajadores administrativos y docentes de la Universidad Autónoma de Aguascalientes.

\section{| | | | | | | | | | | | | | | | | | | | | | | | | | | | | | | | | | | | | | | | | | | | | | | | | | | | | | | | | | | | | | | | | | | | | | | | | | | | | | | | | | | | | | | | | | | | | | | | | | | | | | | | | | | | | ||}

\section{Materiales y métodos}

Se trata de un estudio descriptivo, prospectivo y transversal, realizado en la Universidad Autónoma de Aguascalientes. Se convocó a todo el personal docente y administrativo de la Universidad Autónoma de Aguascalientes, acudiendo en total 59 hombres y 86 mujeres. Se registraron la edad y sexo de cada individuo que participó en el estudio, se realizó exploración física, donde se registraron la talla, el peso, IMC, circunferencia abdominal, frecuencia car- diaca, presión arterial sistólica y diastólica. Se realizaron mediciones de laboratorio de glucosa, colesterol total, triglicéridos y colesterol HDL. Se registraron antecedentes heredofamiliares, personales no patológicos y patológicos. Se utilizó el interrogatorio directo para los datos de identificación, edad y antecedentes de importancia. Los parámetros de laboratorio fueron medidos a partir de muestras de sangre tomadas al momento de las mediciones somatométricas e interrogatorio. 


\section{Criterios para diagnóstico de síndrome metabólico y sus componentes}

La definición propuesta por el grupo del Programa Nacional de Educación en Colesterol y Panel de Tratamiento en el Adulto III (NCEP-ATP III) en $2001^{1}$ incluyen el tener 3 o más de los criterios presentados en la Tabla 1.

\section{Tabla I}

\section{Criterios para diagnóstico de síndrome metabólico del NCEP-ATP III}

\begin{tabular}{|lll|}
\hline Componente & Hombres & Mujeres \\
\hline Obesidad central & Perímetro de cintura $>102 \mathrm{~cm}$ & Perímetro de cintura $>88 \mathrm{~cm}$ \\
\hline Hipertrigliceridemia & Triglicéridos $\geq 150 \mathrm{mg} / \mathrm{dL}$ o medicación específica \\
\hline Disminución de HDL & $\begin{array}{l}\text { Colesterol } \mathrm{HDL}<40 \mathrm{mg} / \mathrm{dL} \text { o } \\
\text { medicación específica }\end{array}$ & $\begin{array}{l}\text { Colesterol } \mathrm{HDL}<50 \mathrm{mg} / \mathrm{dL} \text { o } \\
\text { medicación específica }\end{array}$ \\
\hline Hipertensión arterial & Presión sistólica $\geq 130 \mathrm{mmHg}$ o presión diastólica $\geq 85 \mathrm{mmHg}$ o mediación específica \\
\hline Hiperglucemia & $\begin{array}{l}\text { Glucosa plasmática en ayuno } \geq 110 \mathrm{mg} / \mathrm{dL} \text { o medicación específica o diagnóstico } \\
\text { previo de diabetes mellitus tipo } 2\end{array}$ \\
\hline
\end{tabular}

Tabla 1 Criterios para el diagnóstico de Síndrome metabólico propuestos por el NCEP-ATP III. NCEPATP III: Programa Nacional de Educación en Colesterol y Panel de Tratamiento en el Adulto III. HDL: Lipoproteínas de alta densidad.

La presencia de cada componente del síndrome metabólico fue determinada siguiendo los criterios del ATP III (tabla 1). La presencia de un componente fue determinada según estos parámetros tomando las cifras determinadas en esta tabla para cada sexo. Se utilizó estadística descriptiva para reportar los valores. Se reportan de la forma media \pm desviación estándar. Se realizaron comparaciones para variables categóricas con la prueba de $\chi^{2}$, así como la prueba t de Student para comparar medias de variables cuantitativas entre los sexos. Se consideró $p<0.05$ como un resultado significativo.

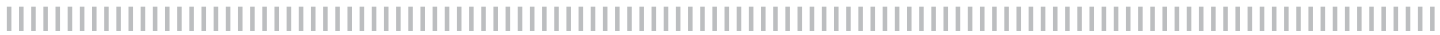

\section{Resultados}

\section{Características demográficas de la población estudiada}

Las principales características demográficas y somatométricas se muestran en la tabla 2. Se puede observar que los grupos de hombres y mujeres fueron similares en cuanto a edad e índice de masa corporal (IMC). La comparación entre talla y peso es, como lo esperado, mayor en el hombre.

\section{Tabla 2}

Promedio de las características demográficas de ambos sexos. Los resultados se presentan como media \pm desviación estándar. IMC: Índice de masa corporal.

\begin{tabular}{|lll|}
\hline Característica & Mujeres & Hombres \\
\hline Edad (años) & $44.2 \pm 10.6$ & $44 \pm 10.4$ \\
\hline Talla $(\mathrm{cm})$ & $158.2 \pm 5.8$ & $172.8 \pm 5.7$ \\
\hline Peso $(\mathrm{kg})$ & $69.3 \pm 13.5$ & $82.2 \pm 12.6$ \\
\hline IMC $(\mathrm{kg} / \mathrm{m} 2)$ & $27.7 \pm 5.5$ & $27.5 \pm 3.8$ \\
\hline
\end{tabular}


Se puede notar que el promedio de IMC de ambos grupos fue mayor a 25 . En las mujeres encontramos que el $34.88 \%$ tiene sobrepeso y el $27.91 \%$ tiene obesidad. En los hombres, estas proporciones fueron de $47.46 \%$ y $23.73 \%$, respectivamente, cifras similares a las presentadas en la Encuesta Nacional de Salud y Nutrición 2012 (Barquera, 2013) ${ }^{13}$.

\section{Antecedentes de los individuos}

Del total de los individuos, $73.1 \%(n=106)$ tenían antecedentes heredofamiliares de diabetes mellitus y $71.72 \% \quad(n=104)$ de hipertensión arterial. El tabaquismo fue poco prevalente, sólo $15.17 \%(n=22)$ individuos fumaban, en su mayoría hombres. Con respecto al alcohol, 30.34\% $(n=44)$ personas refirieron su ingesta frecuente, la mayoría hombres. El 44.14\% $(n=64)$ de los individuos reportaron ser sedentarios, $6.9 \%(n=10)$ tenían diabetes mellitus previamente diagnosticada y $15.17 \% \quad(n=22)$ tenían hipertensión arterial previamente diagnosticada.

\section{Datos bioquímicos y somatométri- cos del síndrome metabólico}

En la tabla 3 se muestran los datos de los promedios de las mediciones más relevantes para el diagnóstico de síndrome metabólico entre mujeres y hombres.

Sólo se obtuvo una comparación significativa entre el nivel de triglicéridos $(p=0.013)$; ya que la medición de la circunferencia abdominal y colesterol HDL se ven influidas por el sexo, no se consideraron estas comparaciones.

\section{Tabla 3}

Promedios de las mediciones bioquímicas y somatométricas principales en ambos sexos. Los resultados se presentan como media \pm desviación estándar.

\begin{tabular}{|lll|}
\hline Característica & Mujeres & Hombres \\
\hline Circunferencia Abdominal $\mathbf{( c m )}$ & $88.9 \pm 12.5$ & $97.4 \pm 10.1$ \\
\hline Presión arterial sistólica $(\mathbf{m m H g})$ & $118.5 \pm 11.8$ & $120.6 \pm 12.8$ \\
\hline Presión arterial diastólica $\mathbf{( m m H g )}$ & $79.3 \pm 10.2$ & $79.6 \pm 10.4$ \\
\hline Glicemia $\mathbf{( m g / d L ) ~}$ & $109.4 \pm 31.1$ & $105 \pm 14.7$ \\
\hline Colesterol total $\mathbf{( m g / d L )}$ & $194.8 \pm 32.6$ & $192.8 \pm 40.9$ \\
\hline Triglicéridos $\mathbf{( m g / d L )}$ & $160.2 \pm 78.8$ & $202.8 \pm 107.7$ \\
\hline Colesterol HDL $\mathbf{( m g / d L )}$ & $52.5 \pm 14.1$ & $43.3 \pm 10.9$ \\
\hline
\end{tabular}

\section{Prevalencia del síndrome metabólico}

La prevalencia de síndrome metabólico, según los criterios del NCEP-ATP III, fue de $36.05 \%$ en las mujeres y de $27.12 \%$ en los hombres, la comparación no fue estadísticamente significativa. En total, la prevalencia de síndrome metabólico fue de $32.41 \%$.

\section{Prevalencia de los componentes del síndrome metabólico en la población estudiada}

La frecuencia relativa de los componentes del síndrome metabólico se muestra en la figura 2. Se puede observar que el perfil de frecuencias es diferente en ambos sexos. Los componentes más frecuentes en el sexo masculino fueron la hipertrigliceridemia y 
la disminución de $\mathrm{HDL}$, presentándose en un $66.1 \%$ y $40.68 \%$, respectivamente. En el sexo femenino, los componentes más frecuentes fueron la disminución en HDL y la obesidad central, con una frecuencia relativa de $51.2 \%$ y $47.67 \%$ respectivamente. Se realizó la comparación de la prevalencia de cada factor entre ambos grupos de sexo y sólo se observó diferencia significativa en la prevalencia de hipertrigliceridemia siendo ésta mayor en el sexo masculino $(66.10 \%$ vs $41.86 \%, p=0.004)$ y en la prevalencia de obesidad central, siendo ésta mayor en el sexo femenino $(47.67 \%$ vs $28.81 \%, p=0.023$ ).

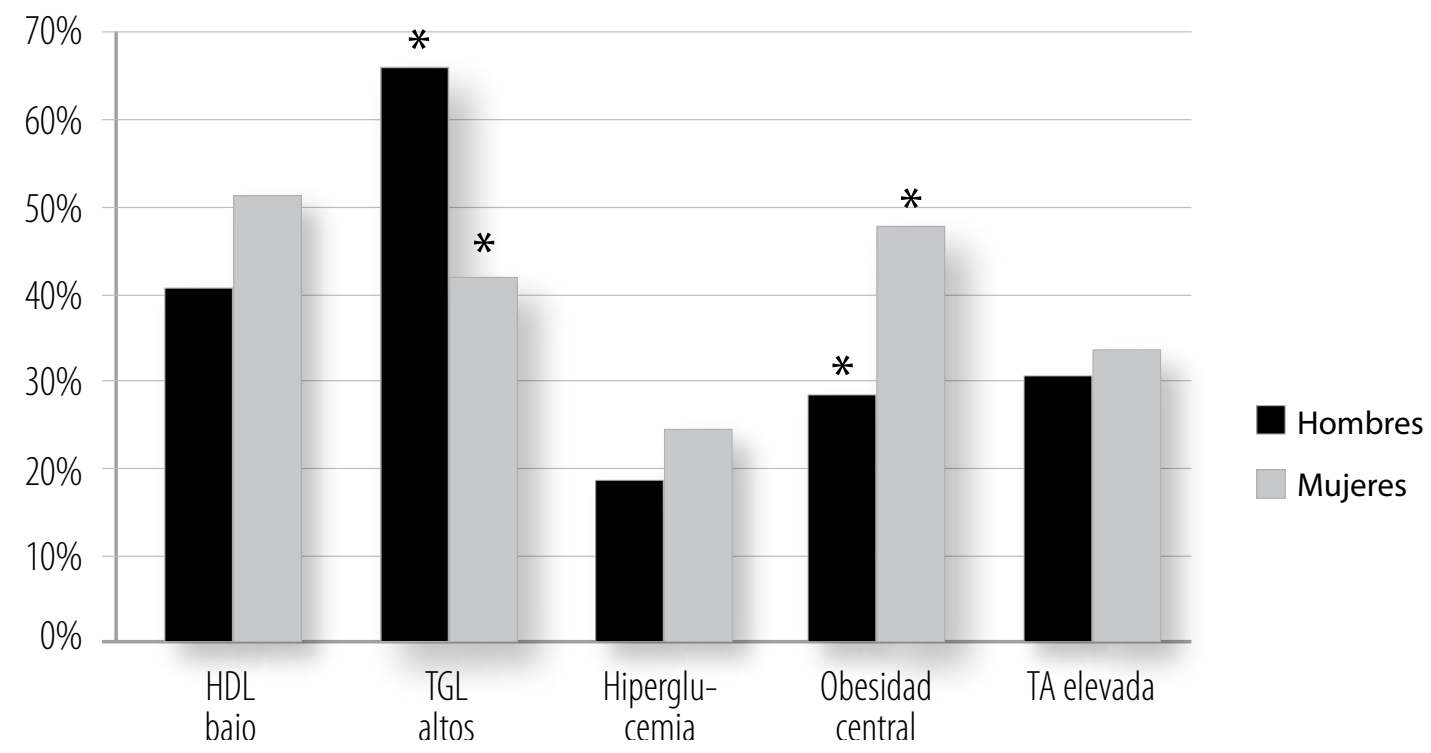

Figura 2: Frecuencia relativa de cada componente del síndrome metabólico en los individuos estudiados. HDL: Lipoproteínas de baja densidad, TGL: Triglicéridos, TA: Tensión arterial, *, **: Comparaciones significativas.

\section{Número de componentes del síndrome metabólico}

De los hombres sólo el $15.25 \%$ y de las mujeres sólo el $19.77 \%$ no tuvieron ningún componente del síndrome metabólico.

\section{Prevalencia de componentes en individuos con síndrome metabólico}

Entre los hombres con síndrome metabólico, los componentes más frecuentes fueron hipertrigliceridemia, hipertensión, y obesidad centrípeta respectivamente (figura 3).Entre las mujeres con síndrome metabólico se observó un perfil distinto.
Los componentes más frecuentes fueron la obesidad centrípeta, disminución de HDL y empatados en tercer lugar la hipertrigliceridemia y la hipertensión (figura 4). Estos datos concuerdan un tanto en los factores más frecuentes en la población general. Es de notar que el componente menos frecuente tanto en la población total, como en los que tuvieron el diagnóstico de síndrome metabólico fue la hiperglucemia, independientemente del sexo. A pesar de que la relación más directa y clásicamente estudiada de la resistencia a la insulina es la hiperglucemia y la diabetes mellitus, este es el factor menos frecuentemente encontra- 
do tanto en la población total como en los tienen una importancia que radica mucho que tienen síndrome metabólico. Así pues, más allá de la diabetes mellitus tipo 2. el síndrome metabólico y sus componentes

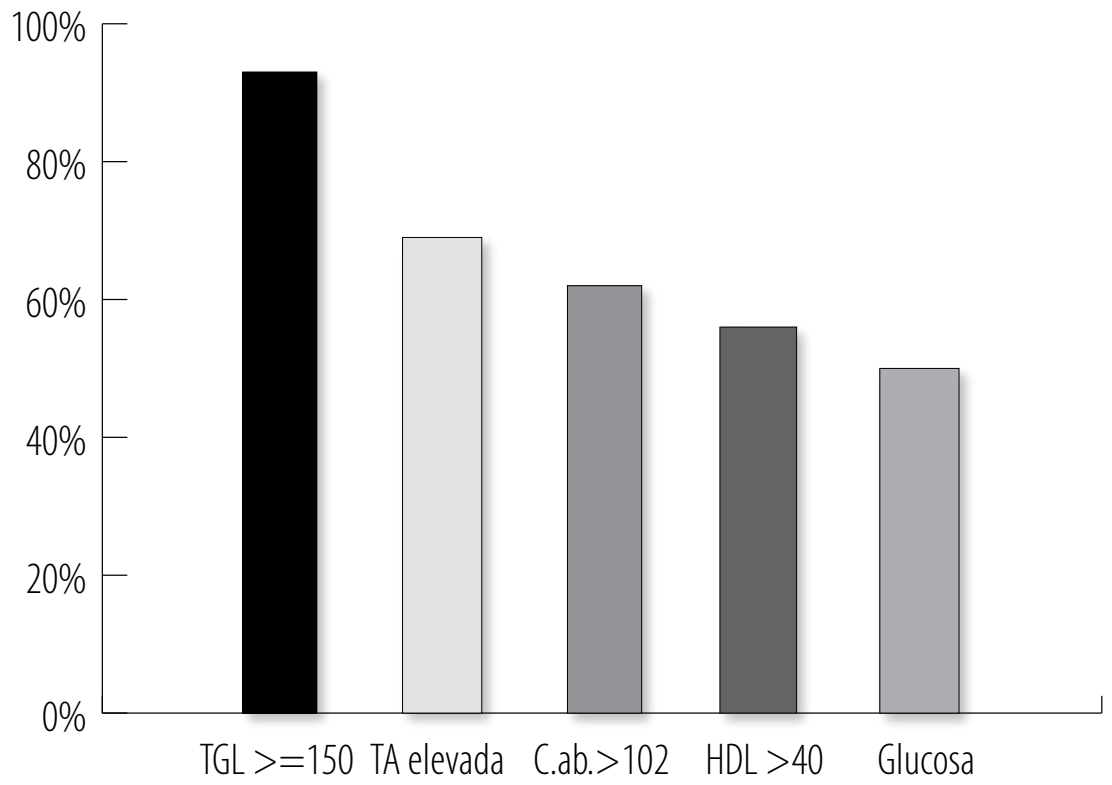

Figura 3: Prevalencia de los componentes de síndrome metabólico en hombres con el diagnóstico. TGL: Triglicéridos, TA: Tensión arterial, C. Ab.: Circunferencia abdominal, HDL: Lipoproteinas de baja densidad.

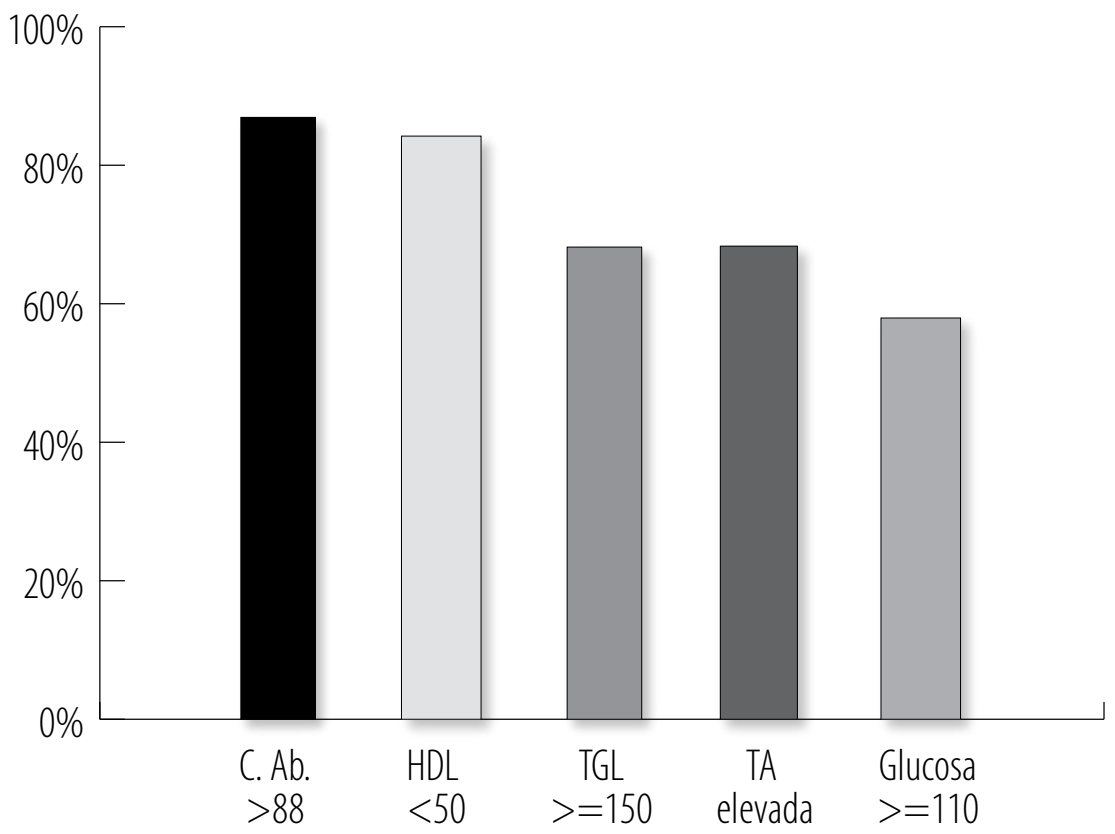

Figura 4: Prevalencia de los componentes de síndrome metabólico en mujeres con el diagnóstico. TGL: Triglicéridos, TA: Tensión arterial, C.Ab.: Circunferencia abdominal, HDL: Lipoproteínas de baja densidad. 


\section{Obesidad centrípeta y obesidad abdominal}

Uno de los componentes centrales del síndrome metabólico es la obesidad central. Como se puede observar por los resultados presentados, en el grupo de mujeres es donde esto cobra especial importancia.

En el grupo de mujeres, se comparó a las pacientes con una circunferencia abdominal mayor a $88 \mathrm{~cm}$ con las que tienen circunferencia abdominal menor o igual a $88 \mathrm{~cm}$. Se halló que el grupo con obesidad centrípeta tiene una prevalencia de síndrome metabólico significativamente mayor a la que no $(65.85 \%$ vs $9.3 \%, p<0.0001)$. Además, tomando a la obesidad centrípeta como prueba diagnóstico, tendría una sensibilidad del $87 \%$ (IC: $75-99 \%$ ) y un valor predictivo negativo de $91 \%$ (IC: $82-99 \%$ ).

El índice de masa corporal mayor a 25 mostró un comportamiento similar en ambos sexos, con una sensibilidad y valor predictivo negativo mayores a los de la circunferencia abdominal mayor a $88 \mathrm{~cm}$ en mujeres, y con sensibilidad de $94 \%$ y valor predictivo negativo de $94 \%$ para la población general tomando en cuenta ambos sexos.
Estos resultados podrían extrapolarse a postular la medición de la circunferencia abdominal y un índice de masa corporal mayor a 25 como pruebas de tamizaje para la detección de síndrome metabólico en mujeres, que aunque poco específica (74\%), es sencilla y muy barata, a diferencia de los exámenes de laboratorio que deben de hacerse para diagnosticar el síndrome metabólico.

\section{Comparación de grupo con síndrome metabólico y grupo sin síndrome metabólico}

Encontramos las medias de edad, peso, índice de masa corporal y componentes del síndrome metabólico mayores en el grupo con el diagnóstico con respecto a los otros en ambos sexos. Podemos observar que, a pesar de eso, la cifra promedio de triglicéridos de los hombres sin síndrome metabólico es bastante más alta que el límite superior normal y que, además, su cifra de HDL no es muy diferente. La edad promedio fue mayor en ambos sexos en el grupo con síndrome metabólico. El promedio de índice de masa corporal de ambos sexos sin síndrome metabólico está por arriba de 25 (tabla 4).

\section{Tabla 4}

Promedios de variables entre hombres y mujeres con y sin síndrome metabólico.

\begin{tabular}{|c|c|c|c|c|}
\hline Característica & $\begin{array}{l}\text { Hombres } \\
\text { con SM }\end{array}$ & $\begin{array}{l}\text { Hombres } \\
\text { sin SM }\end{array}$ & $\begin{array}{l}\text { Mujeres } \\
\text { con SM }\end{array}$ & $\begin{array}{l}\text { Mujeres } \\
\text { sin SM }\end{array}$ \\
\hline Edad (años) & 48.13 & 42.44 & 47.68 & 42.15 \\
\hline Peso (kg) & 90.24 & 79.27 & 76.87 & 64.97 \\
\hline IMC (kg/m2) & 29.98 & 26.6 & 31.14 & 25.81 \\
\hline Circunferencia abdominal (cm) & 104.41 & 94.74 & 97.95 & 83.68 \\
\hline Presión arterial sistólica $(\mathrm{mmHg})$ & 127.19 & 118.12 & 124.83 & 114.98 \\
\hline Presión arterial diastólica (mmHg) & 85.5 & 77.44 & 84.52 & 76.35 \\
\hline Glicemia (mg/dL) & 115 & 101.26 & 126.03 & 99.98 \\
\hline Colesterol total (mg/dL) & 205.44 & 188.14 & 203.39 & 189.91 \\
\hline Triglicéridos (mg/dL) & 228.44 & 193.26 & 205.03 & 134.85 \\
\hline Colesterol HDL (mg/dL) & 42.25 & 43.72 & 45.9 & 56.3 \\
\hline
\end{tabular}




\section{Hiperglucemia y diabetes mellitus previamente diagnosticada}

Aunque la hiperglucemia fue el componente menos frecuente encontrado del síndrome metabólico, tiene relevancia pues se detectaron algunos casos de diabetes mellitus no diagnosticada. En la tabla 5 se muestran los datos de la hiperglucemia y diabetes mellitus.

\section{Tabla 5}

Se muestran los datos de la frecuencia de hiperglucemia, diabetes mellitus y los casos diagnosticados al momento del estudio. DM: Diabetes mellitus

\begin{tabular}{|lccc|}
\hline & Mujeres & Hombres & Todos \\
\hline Número de individuos & 86 & 59 & 145 \\
\hline DM previamente diagnosticada & 7 & 3 & 10 \\
\hline Hiperglucemia & 18 & 9 & 27 \\
\hline DM previamente diagnosticada e hiperglucemia & 6 & 3 & 9 \\
\hline Hiperglucemia sin DM previamente diagnosticada & 12 & 6 & 18 \\
\hline DM recién diagnosticada & 4 & 2 & 6 \\
\hline
\end{tabular}

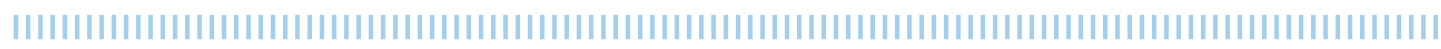

\section{Discusión}

En esta población encontramos prevalencia alta de sobrepeso y obesidad, pero con cifras similares a las reportadas en la Encuesta Nacional en Salud y Nutrición $2012^{13}$. Los antecedentes de diabetes mellitus e hipertensión arterial fueron muy prevalentes, sin embargo, la prevalencia de diabetes mellitus e hipertensión arterial era baja comparada con las cifras nacionales ${ }^{14}$. Cabe destacar que algunas cifras encontradas al momento del estudio eran diagnósticas de diabetes mellitus e hipertensión arterial, aumentando la prevalencia real en la población. Los promedios de los parámetros bioquímicos, en su mayoría, están cercanos o superan los niveles requeridos para considerarlos anormales. Además la prevalencia de ciertos componentes del síndrome metabólico en los sexos excedía el $50 \%$ en algunos casos.

Las diferencias en la fisiopatología, presentación y pronóstico de enfermedades cardiovasculares causadas por el sexo están muy bien descritas en la bibliografía. Si bien existe una variación considerable en la prevalencia del síndrome metabólico dependiendo de la geografía, edad y sexo que puede ser explicada por las diferencias genéticas y factores asociados como patrones dietéticos, físicos, niveles de estrés, estatus socioeconómico y educación, no hay mucha bibliografía que explique y documente las diferencias de presentación y pronóstico del síndrome metabólico en referencia al sexo.

Estudios de la National Health and Nutrition Examination Survey (NHANES) sobre síndrome metabólico encontraron una prevalencia mayor en las mujeres sobre los hombres (22.8 vs $11.2 \%$ ). Otros estudios como el de Miller et. al demostraban que la prevalencia aumentaba al incrementar la edad de la mujer ${ }^{15}$, además encontraron que mujeres con niveles de educación inferior tenían significativamente mayor prevalencia de todos los componentes del síndrome metabólico comparado contra hombres de niveles educacionales similares. La multiparidad, el estrés psicosocial, la pobreza, el desempleo y ser madres solteras predispone a un estilo de vida carencial 
que provoca el desarrollo de anormalidades metabólicas. En países europeos se reporta una prevalencia de síndrome metabólico respecto al sexo de $32.1-42.7 \%$ en mujeres vs el $20.7-37.2 \%$ en hombres. ${ }^{16,17}$

\section{Hipertensión arterial}

La hipertensión arterial es un precursor de enfermedad cardiovascular y es más frecuente en hombres. Sin embargo, los estudios que han evaluado la variabilidad en la incidencia de la hipertensión entre mujeres y hombres han encontrado que los hombres tienden a un aumento de la presión arterial durante y después de la adolescencia mientras que las mujeres demuestran un aumento en la presión arterial en el período posmenopáusico. ${ }^{18}$ En estudios de la NHANES en 1999 a 2004, las mujeres tenían una cifra elevada de presión arterial sistólica y una presión arterial diastólica baja comparadas con los hombres. ${ }^{19} \mathrm{La}$ mayoría de las mujeres hipertensas se encontraba en edad postmenopáusica y la hipertensión estaba asociada a un aumento en la prevalencia de otros factores de riesgo como HDL bajos, colesterol total elevados y obesidad central en la mujer. Dichas diferencias en la presión arterial a lo largo de la vida del hombre y la mujer se explica por parámetros endocrino-biológicos, morfología y distribución del tejido adiposo y mayor rigidez arterial. Estos factores son disminuidos por el efecto hormonal, y una vez que se ven disminuido este efecto protector se da el paso para el desarrollo de la hipertensión sistólica. ${ }^{20}$ Los estrógenos juegan un papel en la regulación del sistema renina-angiotensina-aldosterona y ejercen funciones neuromoduladoras que afectan la actividad simpática. Los estrógenos aumentan la producción de angiotensinógeno y mayor expresión del receptor tipo 2 de la angiotensina, que está asociado a un incremento en la presión arterial, así mismo disminuye la síntesis de renina, enzima convertidora de angiotensina y dis- minuye la expresión el receptor tipo 1 de la Angiotensina. ${ }^{21}$

\section{Resistencia a la insulina}

La forma diagnóstica de la resistencia a la insulina es mediante valores correspondientes de intolerancia a la glucosa y alteraciones de la glucosa en ayuno. En algunos análisis de DECODE y DECODA demostraron que la alteración de la glucosa en ayunas es mayor en hombres que en mujeres de 1.5 a 3 veces. Mientras que la intolerancia a la glucosa fue mayor en mujeres, pero no así en mayores de 80 años. Estas diferencias en el metabolismo de carbohidratos se deben a las diferencias en la masa muscular, tejido adiposo y cantidad hormonal. En resumen las mujeres tienen un porcentaje mayor de obesidad, menos masa muscular, más tejido celular subcutáneo adiposo (localización abdominal y genitofemoral) y una sensibilidad a la insulina más alta. Mientras que los hombres presentan características de más grasa visceral localizada en abdomen y región superior del cuerpo, mayor cantidad de masa muscular y sensibilidad a la insulina mucho menor. ${ }^{22}$

\section{Dislipidemia}

En el estudio MESA, las mujeres mostraron mayores niveles, significativamente, de colesterol total y de HDL, y niveles de LDL y triglicéridos similares a los niveles reportados en los hombres. ${ }^{23}$ Estudios han revelado que las partículas pequeñas de LDL y HDL, así como las partículas grandes de VLDL son las fracciones de lípidos que incrementan el riesgo CVD. Son varios los mecanismos que tratan de explicar dichas situaciones, de diferencia en el perfil lipídico entre las mujeres y los hombres. Que incluyen las diferentes actividades y efectos de la lipasa hepática y lipoproteína lipasa. La lipoproteína lipasa media la captación de triglicéridos por el tejido adiposo. Las mujeres tienen una mayor actividad de 
la lipoproteína lipasa por unidad de tejido adiposo en comparación con los hombres, lo que conduce a metabolismo del colesterol más favorable. El colesterol LDL es un buen predictor de riesgo cardiovascular en mujeres. Mientras que el colesterol noHDL es un mejor predictor en hombres.

\section{Obesidad}

Estudios globales colocan el porcentaje de prevalencia de obesidad en mujeres en un $38 \%$ mientras que en hombres se reporta un $36.9 \% .{ }^{24}$ Las mujeres tienen una prevalencia mayor de sobrepeso y obesidad en países en vías de desarrollo. La circunferencia abdominal parece ser un mejor marcador de riesgo CDV comparado con el BMI. Los patrones del estilo de vida que a su vez se ven influenciados por factores culturales son los principales contribuyentes a esta diferencia significativa de prevalencia de sobrepeso, complementado por los factores genéticos y hormonales.

El tejido adiposo visceral es más común en los hombres y es anatómicamente y metabólicamente diferente al tejido adiposo subcutáneo, más frecuente en mujeres. ${ }^{25}$ La caída de los niveles de estrógenos durante la menopausia ha sido asociada a un incremento en la obesidad visceral y un riesgo cardiovascular mayor.

\section{Conclusiones}

El síndrome metabólico es una entidad frecuente en la población estudiada. El riesgo que conlleva tiene un impacto importante en la morbimortalidad en la población mexicana. Las características del síndrome metabólico son diferentes en hombres y mujeres, posiblemente explicado por las diferencias hormonales entre ambos. En el caso específico de la población estudiada, se encuentra una prevalencia elevada de este síndrome, pero mucho menor a las reportadas en la población mexicana.

El reconocimiento de este síndrome como un problema de salud en nuestra población es importante para poder emprender acciones de prevención primaria y secundaria. Hemos visto recientemente como este problema está comenzando cada vez a edades más tempranas y que son necesarias más acciones de prevención. Si reflexionamos sobre la definición de síndrome metabólico, nos daremos cuenta de que su misma definición nos deja claro que, en sí, no conforma una enfermedad, sino un grupo de factores de riesgo prevenibles, en los cuales hay que intervenir para modificarlos y así modificar el riesgo que conllevan.

En la población estudiada en particular, las propuestas a futuro son el establecimiento de propaganda favoreciendo la actividad física y dieta sana, la implementación de más actividades deportivas para los alumnos y para los trabajadores de la institución, realizar charlas donde se den a conocer lo que es el síndrome metabólico y sus consecuencias.

Debido a las diferencias observadas entre sexos, es posible plantear que la intervención en ambos grupos deba ser distinta. En el sexo masculino enfocada a la disminución de las cifras de triglicéridos por medio de intervenciones en la dieta; mientras en el sexo femenino con intervenciones dietéticas y de actividad física para disminuir la obesidad centrípeta. 


\section{Bibliografía}

1. NCEP - ATP III. (2002). Third Report of the National Cholesterol Education Program (NCEP) Expert Panel on Detection, Evaluation, and Treatment of High Blood Cholesterol in Adults (Adult Treatment Panel III) final report. Circulation. Dec 17;106(25):3143-421.

2. Russo, A., Autelitano, M., \& Bisanti, L. (2008). Metabolic syndrome and cancer risk. European Journal of Cancer, 44(2), 293-297. http://doi.org/10.1016/j. ejca.2007.11.005.

3. Wayburn, E., \& Beckh, W. (1942). Insulin Resistance in Diabetes Mellitus. The Journal of Clinical Endocrinology \& Metabolism, 2(8), 511-518. http://doi. org/10.1210/jcem-2-8-511

4. Molina HS, Pérez IA, Alonso AA, Martínez JPD, Castellanos MP, Laisequilla CF del V, et al. (2015). Carga económica de la obesidad y sus comorbilidades en pacientes adultos en México. PharmacoEconomics Span Res Artic. Sep 17;1-8.

5. Kim J-Y, Mun H-S, Lee BK, Yoon SB, Choi E-Y, Min P-K, et al. (2010) Impact of Metabolic Syndrome and Its Individual Components on the Presence and Severity of Angiographic Coronary Artery Disease. Yonsei Med J. Sep 1;51(5):676-82.

6. Jeppesen J, Hein HO, Suadicani P, Gyntelberg F. (1997) Relation of high TG-low HDL cholesterol and LDL cholesterol to the incidence of ischemic heart disease. An 8-year follow-up in the Copenhagen Male Study. Arterioscler Thromb Vasc Biol. Jun;17(6):111420.

7. Mazza A, Tikhonoff V, Schiavon L, Casiglia E. (2005) Triglycerides + high-density-lipoprotein-cholesterol dyslipidaemia, a coronary risk factor in elderly women: the CArdiovascular STudy in the ELderly. Intern Med J. Oct;35(10):604-10.

8. ISEA. Principales causas de mortalidad general 2010 - 2014 [Internet]. 2015 [citado 2015 Nov 5]. Available from: http://www.aguascalientes.gob.mx/ISEA/ mortgral.asp.

9. INEGI. Mortalidad [Internet]. INEGI. 2013 [cited 2015 Nov 10]. Available from: http://www.inegi.org.mx/ est/contenidos/proyectos/registros/vitales/mortalidad/

10. Olaiz-Fernández G, Rivera-Dommarco J, Shamah-Levy $T$, Rojas R, Villalpando-Hernández $S$, HernándezAvila M, Sepúlveda-Amor J. Encuesta Nacional de Salud y Nutrición 2006. Cuernavaca, México: Instituto Nacional de Salud Pública, 2006.

11. Pedroza-Tobias A, Trejo-Valdivia B, Sanchez-Romero LM, Barquera S. (2014) Classification of metabolic syndrome according to lipid alterations: analysis from the Mexican National Health and Nutrition Survey 2006. BMC Public Health. 14:1056.

12. Alberti $K G M M$, Eckel $R H$, Grundy $S M$, Zimmet $P Z$, Cleeman J, Donato KA, et al. (2009). Harmonizing the Metabolic Syndrome A Joint Interim Statement of the International Diabetes Federation Task Force on Epidemiology and Prevention; National Heart, Lung, and Blood Institute; American Heart Association; World Heart Federation; International Atherosclerosis Society; and International Association for the Study of Obesity. Circulation. Oct 20;120(16):1640-5.

13. Barquera S, Campos-Nonato I, Hernández-Barrera $L$, et al. (2013). Prevalencia de obesidad en adultos mexicanos, ENSANUT 2012. Salud Pública Méx; Vol. 55(sup 2):151-160.

14. Gutiérrez JP, Rivera-Dommarco J, Shamah-Levy $T$, Villalpando-Hernández S, Franco A, Cuevas-Nasu L, Romero-Martínez M, Hernández-Ávila M. Encuesta Nacional de Salud y Nutrición 2012. Resultados Nacionales. Cuernavaca, México: Instituto Nacional de Salud Pública (MX), 2012.

15. Loucks EB, Rehkopf DH, Thurston RC, Kawachi I. Socioeconomic disparities in metabolic syndrome differ by gender: evidence from NHANES III. Ann Epidemiol. 2007;17(1):19 - 26.

16. Mabry RM, Reeves MM, Eakin EG, Owen N. Gender differences in prevalence of the metabolic syndrome in gulf cooperation council countries: a systematic review. Diabet Med. 2010;27(5):593-7.

17. Erem C, Hacihasanoglu A, Deger O, Topbas M, Hosver I, Ersoz HO, et al. Prevalence of metabolic syndrome and associated risk factors among Turkish adults: Trabzon MetS study. Endocrine. 2008;33(1):9 - 20.

18. Levine DA, Lewis CE, Williams OD, Safford MM, Liu $\mathrm{K}$, Calhoun DA, et al. Geographic and demographic variability in 20-year hypertension incidence: the CARDIA study. Hypertension. 2011;57(1):39 - 47.

19. Ong KL, Tso AW, Lam KS, Cheung BM. Gender difference in blood pressure control and cardiovascular risk factors in Americans with diagnosed hypertension. Hypertension. 2008;51(4):1142 - 8.

20. Rossi $P$, Frances $Y$, Kingwell BA, Ahimastos AA. Gender differences in artery wall biomechanical properties throughout life. J Hypertens. 2011;29(6):1023 - 33.

21. Denton KM, Hilliard LM, Tare M. Sex-related differences in hypertension: seek and ye shall find. Hypertension. 2013;62(4): 674- 7 .

22. Varlamov $O$, Bethea $C L$, Roberts Jr CT. Sex-specific differences in lipid and glucose metabolism. Front Endocrinol (Lausanne). 2015;5:241.

23. Goff Jr DC, Bertoni AG, Kramer H, Bonds D, Blu menthal RS, Tsai MY, et al. Dyslipidemia prevalence, treatment, and control in the Multi-Ethnic Study of Atherosclerosis (MESA): gender, ethnicity, and coronary artery calcium. Circulation. 2006;113(5):64756.

24. Ng $M$, Fleming $T$, Robinson $M$, Thomson B, Graetz $\mathrm{N}$, Margono C, et al. Global, regional, and national prevalence of overweight and obesity in children and adults during 1980-2013: asystematic analysis for the global burden of disease study 2013. Lancet. 2014:384(9945):766-81.

25. Pradhan AD. Sex differences in the metabolic syndrome: implications for cardiovascular health in women. Clin Chem. 2014;60(1):44-52. 